\title{
Long Memory from Sauerbrey Equation: A Case in Coated Quartz Crystal Microbalance in terms of Ammonia
}

\author{
Xiaohua Wang, ${ }^{1}{ }^{M i n g ~ L i,}{ }^{1}$ and Shengyong Chen ${ }^{2}$ \\ ${ }^{1}$ School of Information Science and Technology, East China Normal University, No. 500, \\ Dong-Chuan Road, Shanghai 200241, China \\ ${ }^{2}$ College of Computer Science, Zhejiang University of Technology, Hangzhou 310023, China \\ Correspondence should be addressed to Ming Li, ming_lihk@yahoo.com
}

Received 10 November 2010; Accepted 24 November 2010

Academic Editor: Cristian Toma

Copyright (C 2011 Xiaohua Wang et al. This is an open access article distributed under the Creative Commons Attribution License, which permits unrestricted use, distribution, and reproduction in any medium, provided the original work is properly cited.

The Sauerbrey equation is a useful empirical model in material science to represent the dynamics of frequency change denoted by $\Delta f$ in an area, denoted by $A$, of the electrode in terms of the increment of the mass, which is denoted by $\Delta m$, loaded on the surface of the crystal under a certain resonant frequency $f_{0}$. For the purpose of studying $\Delta f$ from the point of view of time series, we first propose two types of the modified representations of the Sauerbrey equation by taking time as an argument to represent $\Delta f$ as a function expressed by $x\left(t, f_{0}, A, \Delta m\right)$, where $t$ is time. Usually, $\Delta f$ is studied experimentally for the performance evaluation of the tested quartz used in ammonia sensors. Its properties in time series, however, are rarely reported. This paper presents the fractal properties of $\Delta f$. We will show that $\Delta f$ is long range dependent (LRD). Consequently, it is heavy tailed according to the Taqqu's theorem. The Hurst parameter $(H)$ of $\Delta f$ approaches one, implying its strong long memory, providing a new explanation of the repeatability of the experiments and novel point of view of the dynamics of $\Delta f$ relating to the Sauerbrey equation in material science.

\section{Introduction}

Ammonia is a type of gas useful for synthesizing various materials in chemical engineering. On the other side, it is a gas harmful to human body. Therefore, the research regarding monitoring the ammonia in different concentrations is desired for atmospheric environmental measurements and control. The ammonia sensor may yet be a desirable device for this purpose; see for example, Wang et al. [1].

In the aspect of ammonia sensing, an interesting phenomenon of the time-dependent frequency increment that is denoted by $\Delta f$, that is, frequency change, responding to the 
coated sensors working under certain humidity was observed in the experimental research by Wang et al. [2]. That phenomenon of the time-dependent $\Delta f$ appears a random pulse series. Nevertheless, it was only qualitatively described in [2]. Its statistical properties remain unknown. This paper aims at revealing the statistical properties of that pulse time series. The contribution points of this paper are in three aspects. First, we will give two types of the modified representations of the Sauerbrey equation towards investigating the dynamics of $\Delta f$ based on time series. Second, we will point out that it is LRD and accordingly it is heavy tailed according to the Taqqu's theorem. Finally, we will show that the value of $H$ of $\Delta f$ is approximately equal to one. Hence, $\Delta f$ relating to the Sauerbrey equation used in the experiments of [2] has strong long-range persistence, which may be served as a new explanation to describe the repeatability of the experiments done in [2].

The rest of the paper is organized as follows. We will give the preliminaries regarding the experiments on the time-dependent $\Delta f$ and propose modifications of the standard Sauerbrey equation in Section 2. The fractal behavior of the pulse phenomenon of $\Delta f$ is explained in Section 3. Discussions are given in Section 4. Finally, Section 5 concludes the paper.

\section{Modified Representations of the Sauerbrey Equation}

In the experiments by Wang et al. [2], $\mathrm{Pd}^{2+}$ doped $\mathrm{ZnO}$ (zinc oxide) nanotetrapods were prepared and studied for the detection of ammonia. The investigated gas sensors were featured by the combination of a quartz crystal microbalance (QCM) as a transducer and $\mathrm{Pd}^{2+}$ doped $\mathrm{ZnO}$ nanotetrapods as a sensing element. The characteristics, including the sensitivity, stability, and reproducibility of the resulted sensors, were studied under different concentration of ammonia in [2].

Note that quartz crystal microbalance (QCM) is an extremely sensitive mass device. The sensing principle of QCM is to transform the mass change into frequency shifts. In the experiments described in [2], $\mathrm{Pd}^{2+}$ doped $\mathrm{ZnO}$ nanotetrapods were put on the QCM; if the resonance frequency of uncoated QCM was recorded, the mass of the $\mathrm{Pd}^{2+}$ doped $\mathrm{ZnO}$ nanotetrapods can be calculated according to the frequency shifts between the uncoated QCM and the coated one. Based on the same principle, if the coated QCM adsorbed ammonia, the mass of ammonia can also be calculated. The measured frequency shifts were used to evaluate the mass change based on the Sauerbrey equation expressed by

$$
\Delta f=-2.26 \times 10^{-6} \frac{f_{0}^{2}}{A} \Delta m,
$$

where $f(\mathrm{MHz})$ is the fundamental frequency of the unloaded piezoelectric crystal, $f_{0}$ is the resonant frequency $(\mathrm{Hz}), \Delta f$ is the frequency change $(\mathrm{Hz}), \Delta m(\mathrm{~g})$ is the mass change loading on the surface of the crystal, and $A\left(\mathrm{~cm}^{2}\right)$ is the surface area of the electrode. Figure 1 indicates the flow chart of the experiments performed in [2]. The derivation from the standard Sauerbrey equation reported in [3] to (2.1) is given in the appendix.

The experiment system in Figure 1 consists of sample gas inlets with valves, a mass flowmeter, a sensing chamber with QCM, a frequency meter, and a computer for data acquisition and analysis. The valve is a switch for opening and closing the gas tunnel. The mass flowmeter was used for testing the gas concentration. The frequency meter was to 


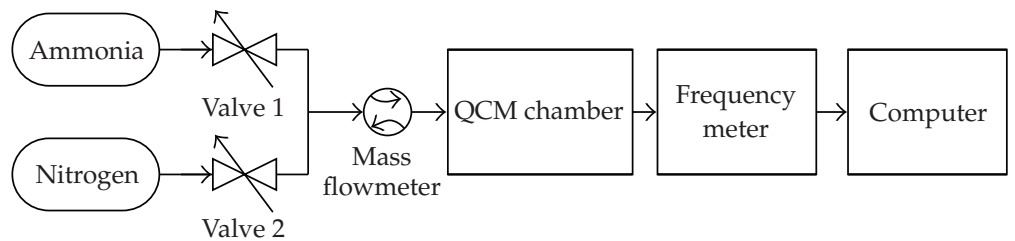

Figure 1: Flow chart of the experiments for obtaining $\Delta f$ for a gas sensing system.

calculate the frequency shifts and send the result to the computer; refer to [2] for the details of the experiments.

In (2.1), $\Delta f$ is in reality a function of time. To clarify this, we express (2.1) by

$$
\Delta f \approx d f=\frac{d f}{d t} d t \approx-2.26 \times 10^{-6} \frac{f_{0}^{2}}{A} d m=-2.26 \times 10^{-6} \frac{f_{0}^{2}}{A} \frac{d m}{d t} d t .
$$

Thus, for a given $n$th round of experiment, we write (2.1) by

$$
y_{n}\left(t, f_{0}, \Delta m, A\right)=-2.26 \times 10^{-6} \frac{f_{0}^{2}}{A} \Delta m, \quad t_{0}<t<t_{0}+T,
$$

where $T$ is the time duration of a round of experiment, $t_{0}$ is the starting time of the $n$th experiment, $y_{n}$ means the frequency increment in the $n$th experiment, and $n$ is a positive integer. We call (2.3) the modified representation of the Sauerbrey equation of type I.

Considering uncertainty in experiments and measurements [4], the result of the $n$th experiment is generally not equal to that of the $(n+1)$ th's. That is,

$$
y_{n} \neq y_{m} \quad \text { for } m \neq n \text {. }
$$

The above expression implies that $y_{n}$ is a random variable. Therefore, we propose the representation of the Sauerbrey equation on the round-by-round basis by

$$
x(t)=y_{n}\left(t, f_{0}, \Delta m, A\right)[u(t)-u(t-n T)], \quad n=1,2, \ldots,
$$

where $u(t)$ is the unit step function. We call (2.5) the modified representation of the Sauerbrey equation of type II. In what follows, $\Delta f$ as well as $x(t)$ are in the sense of (2.5). For convenience, we may write $x(t)$ as

$$
x(t)=x\left(t, f_{0}, \Delta m, A\right) .
$$




\section{Fractal Analysis of the Pulse Phenomenon of Frequency Response to Coated Sensors}

Let $X(t)$ be a second-order stationary random process or random function. Denote by $p(X)$ the probability density function (PDF) of $X(t)$. Then, the probability is given by

$$
P\left(X_{2}\right)-P\left(X_{1}\right)=\operatorname{Prob}\left[X_{1}<\xi<X_{2}\right]=\int_{X_{1}}^{X_{2}} p(\xi) d \xi .
$$

The mean and the autocorrelation function (ACF) of $X(t)$ based on PDF is written by (3.2) and (3.3), respectively,

$$
\begin{gathered}
\mu_{X}=\int_{-\infty}^{\infty} X p(X) d X \\
R_{X}(\tau)=\int_{-\infty}^{\infty} X(t) X(t+\tau) p(X) d X .
\end{gathered}
$$

Let $V_{x}$ be the variance of $X$. Then,

$$
V_{X}=\mathrm{E}\left[X(t)-\mu_{X}\right]^{2}=\int_{-\infty}^{\infty}\left(X-\mu_{X}\right)^{2} p(X) d X
$$

Note 1. If the tail of $p(X)$ is so heavy such that the integrals of (3.2) for $\mu_{X}$ and (3.4) for $V_{X}$ are divergent, we say that $p(\mathrm{X})$ is heavy tailed (Adler et al. [5], Li [6]).

Note 2. If $p(X)$ is heavy tailed, $R_{X}(\tau)$ in (3.3) is slowly decayed. By slowly decayed, we mean that

$$
\int_{-\infty}^{\infty} R_{x}(\tau) d \tau=\infty
$$

Note that (3.5) can be taken as a definition of LRD time series; see for example, Beran [7]. As a matter of fact, according to the Taqqu's theorem, see Abry et al. [8], LRD property of a random function $X(t)$ is a consequence of the heavy-tailed $p(X)(\operatorname{Li}[9])$.

In the Gaussian assumption of $X(t)$, we have

$$
p(X)=\frac{1}{\sqrt{2 \pi V_{X}}} e^{-\left(X-\mu_{X}\right)^{2} / 2 V_{X}} .
$$

In this case, the heavy-tailed $p(X)$ implies that $V_{X} \rightarrow \infty$. In the engineering sense, we do not need infinite variance but $V_{X}$ is large enough, see $\mathrm{Li}$ [10]. 


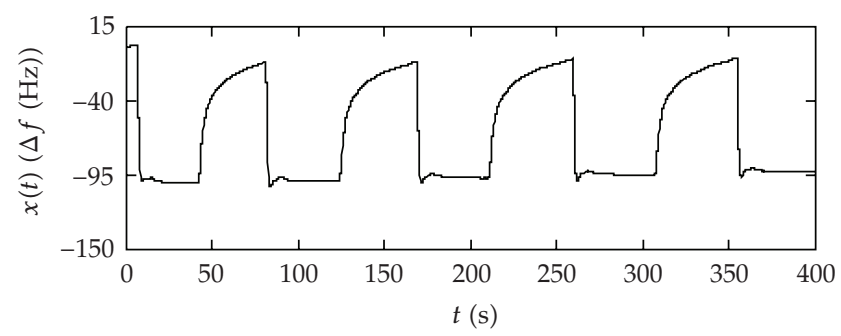

Figure 2: Measured $\Delta f$ of the $\mathrm{Pd}$-doped $\mathrm{ZnO}$ nanotetrapods to the concentration of $\mathrm{NH}_{3}$ from 0 to $240 \mathrm{ppm}$ in time.

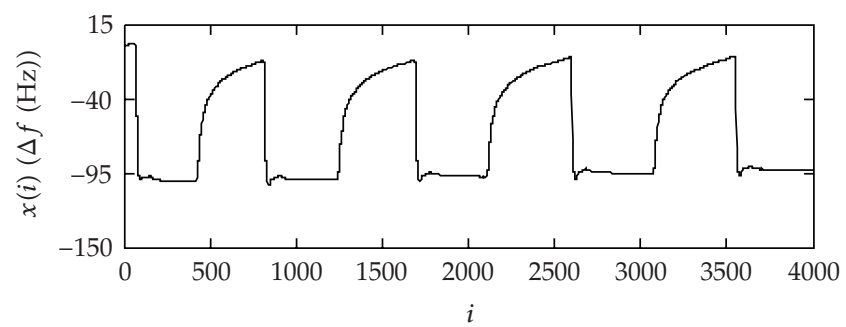

Figure 3: Measured $\Delta f$ of the $\mathrm{Pd}$-doped $\mathrm{ZnO}$ nanotetrapods to the concentration of $\mathrm{NH}_{3}$ from 0 to $240 \mathrm{ppm}$ in sampling index.

Note that $\Delta f$ in the experiments was sampled in the discrete case. Therefore, without loss of generality, we denote $\Delta f$ by

$$
\Delta f=x\left(t, f_{0}, A, \Delta m\right)=x\left(t_{i}\right)
$$

where $x\left(t_{i}\right)$ represents the value of $\Delta f$ of the $i$ th sample, and $i$ is the sample index. Figure 2 indicates the series of $x\left(t_{i}\right)$, where the vertical coordinates is indicated by $x(t)$ and abscissa axis by $t$ for short. Figure 2 is actually a curve of random pulse series representing the timecycling responses of the $\mathrm{Pd}$-doped $\mathrm{ZnO}$ nanotetrapods for the concentration of ammonia gas from 0 to $240 \mathrm{ppm}$. The ratio $\mathrm{Pd}^{2+} / \mathrm{ZnO}$ is $0.04: 1$ in Moore quality. In Figure 2, each pulse stands for the result of a round of experiment. For instance, the second pulse comes from the result of the second round of experiment for $x(t)$. From Figure 2, one sees that the sensor performed in a reproducible manner but random in nature. The experiment was repeated over 20 times and different dosage of Pd. The pulse series indicated in Figure 2 exhibits that $\Delta f$ is random in terms of pulse width, pulse amplitude, and transition time of pulse, as one can see by eye. The random behavior of the pulse series is the focus we work on in this paper. Without loss of generality, we let $x(i)=x\left(t_{i}\right)$, where $i=1, \ldots, 400$. Then, we replot Figure 2 by Figure 3.

Taking into account the random pulse width, pulse amplitude, and pulse transition, we say that $x(i)$ is a random function. By numeric computations, we obtain the ACF of $\Delta f$ as indicated in Figure 4, which implies the remark below.

Remark 3.1. The random function $x(i)$, that is, $\Delta f$, which we investigated, is LRD. In other words, the ACF is slowly decayed such that (3.5) holds. 


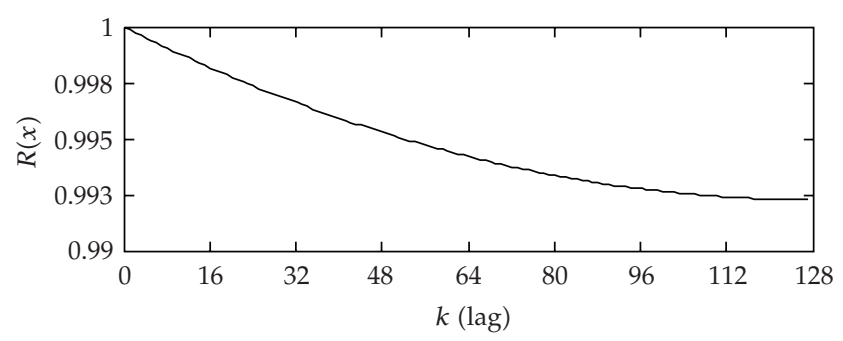

Figure 4: ACF of $x(i)$.

According to the Taqqu's theorem, therefore, comes the following remark.

Remark 3.2. The random function $\Delta f$ described by (2.1) is heavy tailed.

Remark 3.1 implies that the ACF of $x(i)$ has the asymptotic expression given by

$$
R(k) \sim c k^{-b} \quad(k \longrightarrow \infty), b \in(0,1),
$$

where $c>0$ can be either a constant or a slowly varying function.

Denote by $S_{x}(\omega)$ the power spectrum density (PSD) of $x(i)$, where $\omega$ is the angular frequency. Then,

$$
S_{x}(\omega)=\int_{-\infty}^{\infty} R_{x}(\tau) e^{-j \omega \tau} d \tau
$$

Since (3.5) holds, $S_{x}(\omega)$ has to be considered in the domain of generalized functions. According to the Fourier transform in the domain of generalized functions (Kanwal [11], Li and Lim [12]), we immediately obtain

$$
\mathrm{F}\left(|k|^{-b}\right)=2 \sin \left(\frac{\pi b}{2}\right) \Gamma(1-b)|\omega|^{b-1}
$$

where F stands for the operator of the Fourier transform. Therefore, for the measured $\Delta f$, we have the asymptotic expression of the PSD of $x(i)$ below:

$$
\mathrm{F}[R(k)] \sim|\omega|^{b-1} \quad \text { for } \omega \longrightarrow 0 .
$$

Hence, we have the following remark. We note that $\omega$ in (3.9), (3.10), and (3.11) is the angular frequency, which is an argument in the Fourier transform of the ACF of $x(i)$, while $x(i)$ stands for the frequency increment in the Sauerbrey equation. That is, the frequency in $S_{x}(\omega)$ differs in meaning from that in the Sauerbrey equation.

Remark 3.3. The measured $\Delta f$, that is, $x(i)$, is in the class of $1 / f$ noise. 


\section{Discussions}

The previous discussions exhibit three properties of $x(i)$ from a view of fractals. They are heavy-tailed PDF, slowly decayed ACF, and $1 / f$ noise type PSD. Considering the Hurst parameter, we have

$$
H=1-\frac{b}{2}
$$

which characterizes the LRD of $x(i)$ from a view of fractals. By the least square fitting, for the curve in Figure 4, we have

$$
H \approx 1 .
$$

Therefore, the frequency change of the Pd-doped $\mathrm{ZnO}$ nanotetrapods to the concentration of $\mathrm{NH}_{3}$ from 0 to $240 \mathrm{ppm}$ has strong LRD. This implies that the result from the $n$th experiment is strongly correlated to that from the $(n+k)$ th experiment, even if $k$ is large. Hence, we hereby provide, from the point of view of fractals, a quantitative explanation of repeatability of the experiments that were qualitatively stated in [2].

We note that this paper does not say that $x(i)$ obeys the widely used fractal time series model, we mean, fractional Gaussian noise (fGn). In addition, we did not claim anything about the fractal dimension of $x(i)$ either. In the future, we will work on the accurate ACF and the fractal dimension of $x(i)$. To the best of our knowledge, the fractal properties described above may yet imply a considerable advance in the field regarding the frequency change of the Pd-doped $\mathrm{ZnO}$ nanotetrapods to the concentration of $\mathrm{NH}_{3}$ from 0 to $240 \mathrm{ppm}$. Further, we will investigate such a type of random pulses with the differential equations as reported in [13-17]. Finally, we note that $x(i)$ appears deterministic but we did fractal analysis about it to provide an explanation of the uncertainty principle in measurements.

\section{Conclusions}

We have processed the real data of the frequency change of the Pd-doped $\mathrm{ZnO}$ nanotetrapods to the concentration of $\mathrm{NH}_{3}$ from 0 to $240 \mathrm{ppm}$. The present results exhibit that such a pulse series is heavy tailed and has strong LRD. We have also explained the repeatability of the experiments from a view of fractals.

\section{Appendix}

\section{Derivation of $(2.1)$}

The theory of the quartz crystal microbalance (QCM) is the piezoelectric qualities of quartz crystals. The application of an electric field to the electrode of the quartz crystals causes a shear deformation (parallel to the electrode surface). The crystal can be made to resonate if an alternating electric field is applied at a particular frequency $f_{0}$. Deposition of the working electrode layer dampens this resonant frequency. The Sauerbrey equation relates the dampening of frequency, $\Delta f_{0}$, to the change in surface attached mass $\Delta m$. 
The Sauerbrey equation was first introduced by Sauerbrey in [3]. Its standard form is given by

$$
\Delta f=\frac{-2 \Delta m f_{0}^{2}}{A \sqrt{\rho_{q} \mu_{q}}}=-\frac{2 f_{0}^{2}}{A \sqrt{\rho_{q} \mu_{q}}} \Delta m
$$

where $f_{0}$ is the resonant frequency $(\mathrm{Hz}), \Delta f$ is the frequency change $(\mathrm{Hz}), \Delta m$ is the mass change $(g), A$ is the piezoelectrically active crystal area $\left(\mathrm{cm}^{2}\right)$ between electrodes, $\rho_{q}$ is the density of quartz, and $\mu_{q}$ is the shear modulus of quartz for AT-cut crystal. In the experiments described in [2],

$$
\begin{aligned}
& \rho_{q}=2.648 \mathrm{~g} / \mathrm{cm}^{3}, \\
& \mu_{q}=2.947 \times 10^{11} \mathrm{~g} / \mathrm{cms}^{2} .
\end{aligned}
$$

Replacing $\rho_{q}$ and $\mu_{q}$ in (A.1) with (A.2) produces $\Delta f=-2.26 \times 10^{-6}\left(f_{0}^{2} / A\right) \Delta m$, which is the expression (2.1).

\section{Acknowledgments}

This work was partly supported by the National Natural Science Foundation of China (NSFC) under the project Grant nos. 60573125, 60873264, 61070214, and 60870002, the 973 plan under the Project no. 2011CB302800/2011CB302802, NCET, and the Science and Technology Department of Zhejiang Province (2009C21008, 2010R10006, 2010C33095, and Y1090592).

\section{References}

[1] Y. D. Wang, X. H. Wu, Q. Su, Y. F. Li, and Z. L. Zhou, "Ammonia-sensing characteristics of Pt and $\mathrm{SiO}_{2}$ doped $\mathrm{SnO}_{2}$ materials," Solid-State Electronics, vol. 45, no. 2, pp. 347-350, 2001.

[2] X. Wang, J. Zhang, Z. Zhu, and J. Zhu, "Effect of $\mathrm{Pd}^{2+}$ doping on $\mathrm{ZnO}$ nanotetrapods ammonia sensor," Colloids and Surfaces A, vol. 276, no. 1-3, pp. 59-64, 2006.

[3] G. Sauerbrey, "Verwendung von Schwingquarzen zur Wägung dünner Schichten und zur Mikrowägung," Zeitschrift für Physik, vol. 155, no. 2, pp. 206-222, 1959.

[4] J. S. Bendat and A. G. Piersol, Random Data: Analysis and Measurement Procedure, John Wiley \& Sons, 3rd edition, 2000.

[5] R. J. Adler, R. E. Feldman, and M. S. Taqqu, A Practical Guide to Heavy Tails, Birkhauser, 1998.

[6] M. Li, "Fractal time series-a tutorial review," Mathematical Problems in Engineering, vol. 2010, Article ID 157264, 26 pages, 2010.

[7] J. Beran, Statistics for Long-Memory Processes, vol. 61 of Monographs on Statistics and Applied Probability, Chapman and Hall, New York, NY, USA, 1994.

[8] P. Abry, P. Borgnat, F. Ricciato, A. Scherrer, and D. Veitch, "Revisiting an old friend: on the observability of the relation between long range dependence and heavy tail," Telecommunication Systems, vol. 43, no. 3-4, pp. 147-165, 2010.

[9] M. Li, "Fractional Gaussian noise and network traffic modeling," in Proceedings of the 8th WSEAS International Conference on Applied Computer and Applied Computational Science, pp. 34-39, Hangzhou, China, May 2009.

[10] M. Li, "Generation of teletraffic of generalized Cauchy type," Physica Scripta, vol. 81, no. 2, Article ID 025007, 10 pages, 2010. 
[11] R. P. Kanwal, Generalized Functions: Theory and Applications, Birkhäuser, Boston, Mass, USA, 3rd edition, 2004.

[12] M. Li and S. C. Lim, "Power spectrum of generalized Cauchy process," Telecommunication Systems, vol. 43, no. 3-4, pp. 219-222, 2010.

[13] M. Scalia, G. Mattioli, and C. Cattani, "Analysis of large-amplitude pulses in short time intervals: application to neuron interactions," Mathematical Problems in Engineering, vol. 2010, Article ID 895785, 2010.

[14] G. Toma, "Specific differential equations for generating pulse sequences," Mathematical Problems in Engineering, vol. 2010, Article ID 324818, 11 pages, 2010.

[15] E. G. Bakhoum and C. Toma, "Relativistic short range phenomena and space-time aspects of pulse measurements," Mathematical Problems in Engineering, vol. 2008, Article ID 410156, 2008.

[16] C. Cattani and A. Kudreyko, "Application of periodized harmonic wavelets towards solution of eigenvalue problems for integral equations," Mathematical Problems in Engineering, vol. 2010, Article ID 570136, 8 pages, 2010.

[17] C. Cattani and A. Kudreyko, "Harmonic wavelet method towards solution of the Fredholm type integral equations of the second kind," Applied Mathematics and Computation, vol. 215, no. 12, pp. 4164-4171, 2010. 


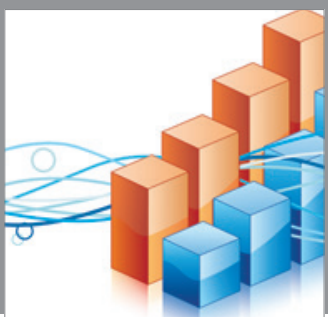

Advances in

Operations Research

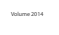

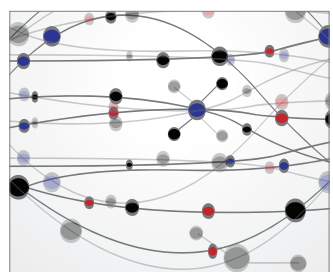

\section{The Scientific} World Journal
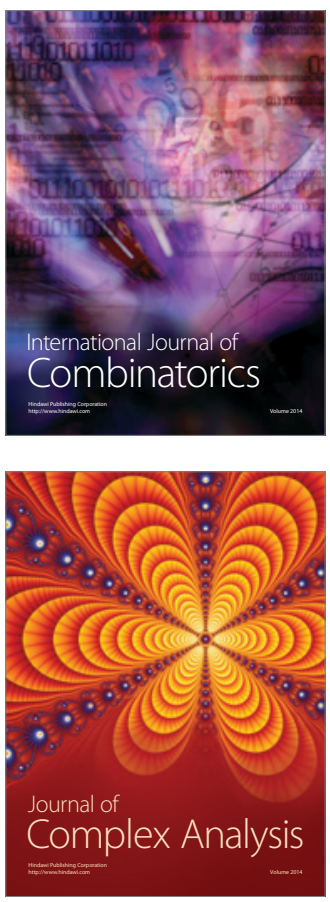

International Journal of

Mathematics and

Mathematical

Sciences
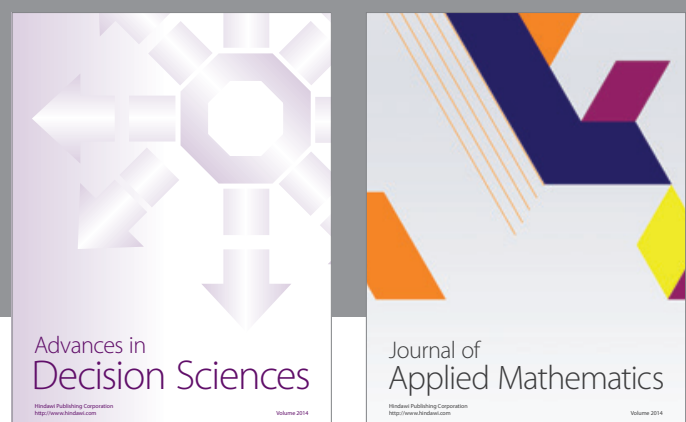

Journal of

Applied Mathematics
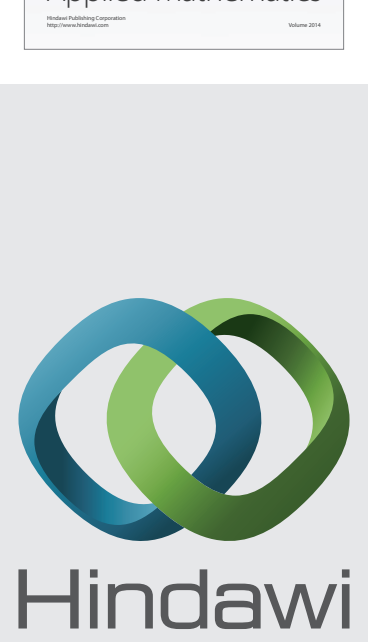

Submit your manuscripts at http://www.hindawi.com
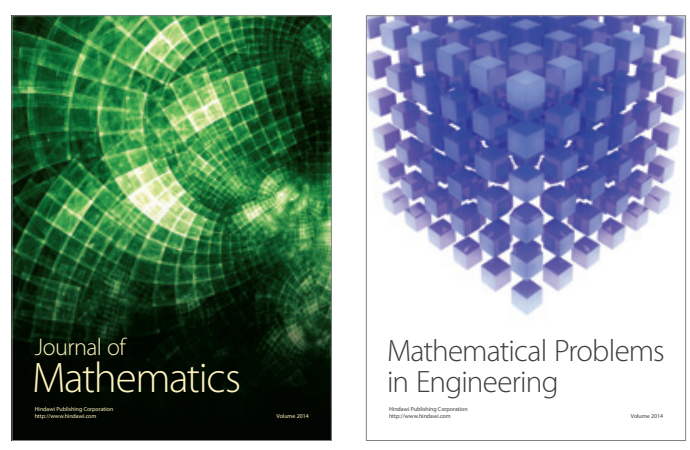

Mathematical Problems in Engineering
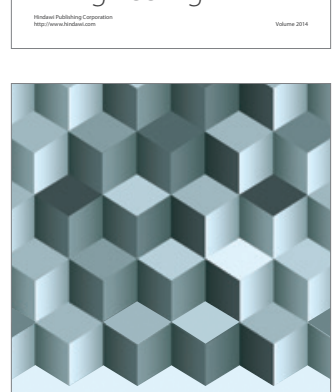

Journal of

Function Spaces
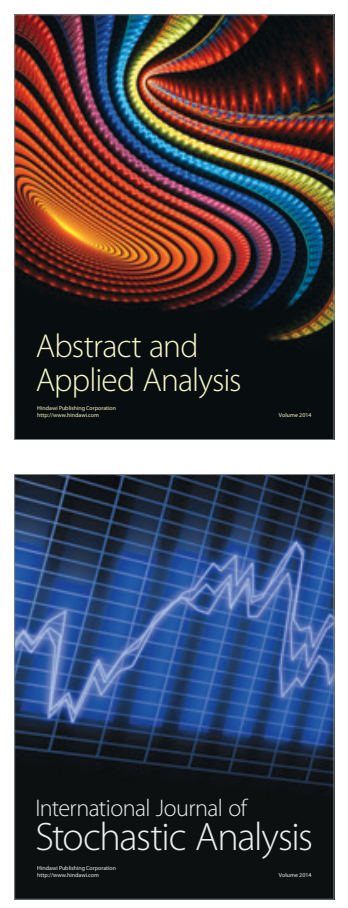

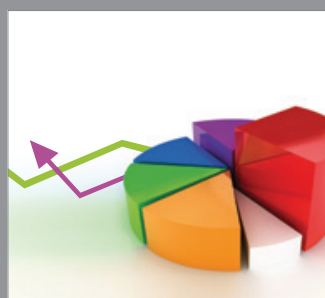

ournal of

Probability and Statistics

Promensencen
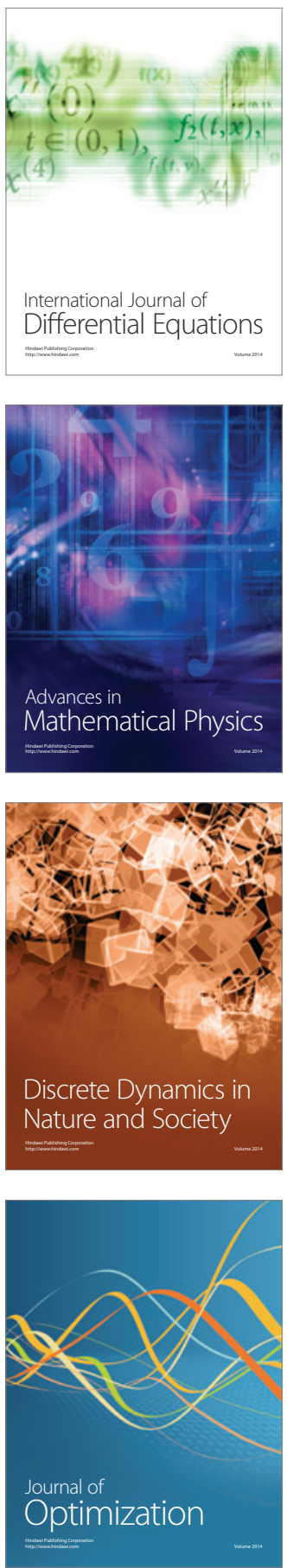